\title{
Lowering the barrier
}

Nano Lett. http://doi.org/cn3z (2018)

Two-dimensional black phosphorus is an attractive material for building electronic devices, such as transistors, because of its inherent bandgap and its potentially high carrier mobility. Transistors made of black phosphorus typically exhibit ambipolar electrical transport, which means they are capable of conducting both electrons and holes. However, for low-power applications it is valuable to have unipolar n- and p-type transistors. Philip Wong and colleagues at Stanford University have now built highperformance unipolar n-type transistors that are based on black phosphorus.

The researchers used the metals erbium and scandium, which have low work functions, as contacts for the black phosphorus transistors. The electrical behaviour of the devices displayed a transition from ambipolar to unipolar after a month at room temperature, which was found to be due to the formation of a metal oxide layer at the contacts. In particular, the metal oxide lowers the height of the energy barrier between the metal contact and the black phosphorus, as well as acting as a doping layer. This enhances the transport of electrons and, as a result, the transistors showed a high n-type current.

Additional analysis showed that extended air exposure degraded the performance of the devices because it oxidizes both the contacts and the black phosphorus layer.

Christiana Varnava

Published online: 14 May 2018

https://doi.org/10.1038/s41928-018-0080-6 